\title{
Stability of intuitionistic fuzzy nonlinear fractional differential equations
}

\author{
Said Melliani ${ }^{1}$, Ali El Mfadel ${ }^{2}$, Lalla Saadia Chadli ${ }^{3}$ \\ and M'hamed Elomari ${ }^{4}$ \\ ${ }^{1}$ LMACS, Sultan Moulay Slimane University \\ BP 523, 23000, Beni Mellal, Morocco \\ e-mail: s.melliani@usms.ma \\ ${ }^{2}$ LMACS, Sultan Moulay Slimane University \\ BP 523, 23000, Beni Mellal, Morocco \\ e-mail: elmfadelali@gmail.com \\ ${ }^{3}$ LMACS, Sultan Moulay Slimane University \\ BP 523, 23000, Beni Mellal, Morocco \\ e-mail: sa.chadli@yahoo.fr \\ ${ }^{4}$ LMACS, Sultan Moulay Slimane University \\ BP 523, 23000, Beni Mellal, Morocco \\ e-mail: m.elomari@usms.ma
}

Received: 25 July 2020

Accepted: 15 December 2020

\begin{abstract}
In this paper, we study the existence and uniqueness results of solution for the intuitionistic fuzzy nonlinear fractional differential equations involving the Caputo concepts of fractional derivative. In addition, we establish essentially the Mittag-Leffler stability result for the intuitionistic fuzzy nonlinear fractional differential equations by giving some sufficient criteria to guarantee the stability of the zero solution. Finally, some examples are presented to illustrate the proposed stability theorem.
\end{abstract}

Keywords: Intuitionistic fuzzy numbers, Intuitionistic fuzzy fractional integral, Intuitionistic fuzzy fractional Caputo derivative, Mittag-Leffler stability.

2020 Mathematics Subject Classification: 34A07. 


\section{Introduction}

Recently, the theory of fuzzy fractional differential equations was proposed to handle uncertainty due to incomplete information that appears in many mathematical or computer models of some deterministic real-world phenomena. This theory was initiated by Agarwal [1] who proposed the concept of solutions for fractional differential equations with uncertainty. Arshad and Lupulescu in [5] proved some results on the existence and uniqueness of solution for the fuzzy fractional differential equations. Later, Alikhani in [3] has proved the existence and uniqueness results for nonlinear fuzzy fractional integral and integrodifferential equations by using the method of upper and lower solutions.

The authors in $[4,21]$ discussed the concepts about generalized Hukuhara fractional RiemannLiouville and Caputo differentiability of fuzzy-valued functions and the equivalence between fuzzy fractional differential equation and fuzzy fractional integral equation is discussed in [11]. In this note and by using the work of Diethelm [9], we study the existence and uniqueness results for the intuitionistic fuzzy nonlinear fractional differential equation.

Stability analysis is also one of the most important issues for differential equations, although this problem has been investigated over many years. Recently, stability of fractional differential systems has attracted increasing interest. The earliest study on stability of fractional differential equations started in [17]. For more details about the stability results and the methods available to analyze the stability of fractional differential equations, the reader may refer to the recent papers $[2,8,20]$ and the references therein.

For the nonlinear fractional differential systems in the classical case, stability analysis is much more difficult than Lyapunov stability $[9,15]$. Applying Lyapunov's direct method for a given nonlinear system means to search for an appropriate Lyapunov function to conclude the stability for this system. A few results are available in $[13,14,16]$. Motivated by Li in [16], in this paper, we present the stability of solution for the intuitionistic fuzzy nonlinear fractional differential equations with the Caputo derivative by using notions of Mittag-Leffler stability.

Our paper is organized as follows: Section 2 gives some basic definitions, lemmas and theorems as preliminaries of intuitionistic fuzzy sets theory. The existence and uniqueness results are given in Section 3, and finally in Section 4 we introduce the concept of Mittag-Leffler stability of the trivial solution of our nonlinear intuitionistic fuzzy system.

\section{Preliminaries}

The concept of fuzzy sets was introduced by Zadeh [26]. Atanassov generalized this idea to intuitionistic fuzzy sets (IFSs) [6], and later there has been much progress in the study of IFSs. As a special case of intuitionistic fuzzy sets, intuitionistic fuzzy number was introduced by Xu [25].

We denote by

$$
\mathbb{I F}(\mathbb{R})=\left\{\langle u, v\rangle: \mathbb{R} \longrightarrow[0,1]^{2}, \quad 0 \leq u(x)+v(x) \leq 1\right\} .
$$

Definition 2.1. [19] An element $\langle u, v\rangle \in \mathbb{I F}(\mathbb{R})$ is called an intuitionistic fuzzy nomber if it satisfies the following conditions: 
1. $\langle u, v\rangle$ is normal, i.e., there exist $x_{0}, x_{1} \in \mathbb{R}$ such that $u\left(x_{0}\right)=1$ and $u\left(x_{1}\right)=1$.

2. $u$ is fuzzy convex and $v$ is fuzzy concave.

3. $u$ is upper semi-continuous and $v$ is lower semi-continuous.

4. $\sup \langle u, v\rangle=\{\overline{x \in \mathbb{R}: v(x)<1}\}$ is bounded.

We denote by $\mathbb{I F}^{1}$ the collection of all intuitionistic fuzzy numbers.

Let $\alpha \in[0,1]$ and $\langle u, v\rangle \in \mathbb{F}^{1}$, we define the upper and lower $\alpha$-cuts of $\langle u, v\rangle$, respectively, by

$$
[\langle u, v\rangle]^{\alpha}=\{x \in \mathbb{R}: v(x) \leq 1-\alpha\}
$$

and

$$
[\langle u, v\rangle]_{\alpha}=\{x \in \mathbb{R}: u(x) \geq \alpha\} .
$$

We define also the intuitionistic fuzzy zero, denoted by $0_{\mathbb{I F}} \in \mathbb{I F}^{1}$, as follows:

$$
0_{\mathbb{I F}}(t)= \begin{cases}(1,0), & \text { if } t=0 \\ (0,1), & \text { if } t \neq 0\end{cases}
$$

Definition 2.2. [19] Let $\left\langle u_{1}, v_{1}\right\rangle,\left\langle u_{2}, v_{2}\right\rangle \in \mathbb{I F}^{1}, \lambda \in \mathbb{R}$ and $\alpha \in[0,1]$, then:

1. $\left(\left\langle u_{1}, v_{1}\right\rangle \oplus\left\langle u_{2}, v_{2}\right\rangle\right)(z)=\left(\sup _{z=x+y} \min \left(u_{1}(x), u_{2}(y)\right), \inf _{z=x+y} \max \left(u_{1}(x), u_{2}(y)\right)\right)$;

2. $\lambda\left\langle u_{1}, v_{1}\right\rangle=\left\langle\lambda u_{1}, \lambda v_{1}\right\rangle$ if $\lambda \neq 0$;

3. $\lambda\left\langle u_{1}, v_{1}\right\rangle=0_{I F}$ if $\lambda=0$;

4. $\left[\left\langle u_{1}, v_{1}\right\rangle \oplus\left\langle u_{2}, v_{2}\right\rangle\right]^{\alpha}=\left[\left\langle u_{1}, v_{1}\right\rangle\right]^{\alpha}+\left[\left\langle u_{2}, v_{2}\right\rangle\right]^{\alpha}$;

5. $\left[\left\langle u_{1}, v_{1}\right\rangle \oplus\left\langle u_{2}, v_{2}\right\rangle\right]_{\alpha}=\left[\left\langle u_{1}, v_{1}\right\rangle\right]_{\alpha}+\left[\left\langle u_{2}, v_{2}\right\rangle\right]_{\alpha} ;$

6. $\left[\lambda\left\langle u_{1}, v_{1}\right\rangle\right]^{\alpha}=\lambda\left[\left\langle u_{1}, v_{1}\right\rangle\right]^{\alpha}$;

7. $\left[\lambda\left\langle u_{1}, v_{1}\right\rangle\right]_{\alpha}=\lambda\left[\left\langle u_{1}, v_{1}\right\rangle\right]_{\alpha}$.

Let $\langle u, v\rangle \in \mathbf{I F}^{1}$ and $\alpha \in[0,1]$, then we define the following sets:

$$
\begin{aligned}
& {[\langle u, v\rangle]_{l}^{+}(\alpha)=\inf \{x \in \mathbb{R}: u(x) \geq \alpha\},} \\
& {[\langle u, v\rangle]_{r}^{+}(\alpha)=\sup \{x \in \mathbb{R}: u(x) \geq \alpha\},} \\
& {[\langle u, v\rangle]_{l}^{-}(\alpha)=\inf \{x \in \mathbb{R}: v(x) \leq 1-\alpha\},} \\
& {[\langle u, v\rangle]_{r}^{-}(\alpha)=\sup \{x \in \mathbb{R}: v(x) \leq 1-\alpha\} .}
\end{aligned}
$$

Remark 2.3. Let $\langle u, v\rangle \in \mathbb{I F}^{1}$ and $\alpha \in[0,1]$, then we have:

$$
\begin{aligned}
& {[\langle u, v\rangle]^{\alpha}=\left[[\langle u, v\rangle]_{l}^{-}(\alpha),[\langle u, v\rangle]_{r}^{-}(\alpha)\right],} \\
& {[\langle u, v\rangle]_{\alpha}=\left[[\langle u, v\rangle]_{l}^{+}(\alpha),[\langle u, v\rangle]_{r}^{+}(\alpha)\right] .}
\end{aligned}
$$

Definition 2.4. [19] Let $\langle u, v\rangle \in \mathbb{I F}^{1}$ and $\alpha \in[0,1]$, we define the diameter of upper and lower $\alpha$-cuts of $\langle u, v\rangle$, respectively, as follows:

$$
\begin{aligned}
& d\left([\langle u, v\rangle]^{\alpha}\right)=[\langle u, v\rangle]_{r}^{-}(\alpha)-[\langle u, v\rangle]_{l}^{-}(\alpha), \\
& d\left([\langle u, v\rangle]_{\alpha}\right)=[\langle u, v\rangle]_{r}^{+}(\alpha)-[\langle u, v\rangle]_{l}^{+}(\alpha) .
\end{aligned}
$$


Proposition 2.5. Let $\alpha, \beta \in[0,1]$ and $\langle u, v\rangle \in \mathbb{I F}^{1}$, then:

1. $[\langle u, v\rangle]_{\alpha} \subset[\langle u, v\rangle]^{\alpha}$;

2. $[\langle u, v\rangle]_{\alpha} e t[\langle u, v\rangle]^{\alpha}$ are nonempty compact convex sets;

3. if $\alpha \leq \beta$, then $[\langle u, v\rangle]^{\beta} \subset[\langle u, v\rangle]^{\alpha}$ and $[\langle u, v\rangle]_{\beta} \subset[\langle u, v\rangle]_{\alpha}$;

4. if $\alpha_{n} \nearrow \alpha$, then $[\langle u, v\rangle]^{\alpha}=\cap_{n}[\langle u, v\rangle]^{\alpha_{n}}$ and $[\langle u, v\rangle]_{\alpha}=\cap_{n}[\langle u, v\rangle]_{\alpha_{n}}$.

Conversely, let $\alpha \in[0,1]$, we put

$$
M_{\alpha}=\{x \in \mathbb{R}: u(x) \geq \alpha\}
$$

and

$$
M^{\alpha}=\{x \in \mathbb{R}: v(x) \leq 1-\alpha\} .
$$

Lemma 2.6. [19] Let $\left\{M^{\alpha}: \alpha \in[0,1]\right\}$ and $\left\{M_{\alpha}: \alpha \in[0,1]\right\}$ be two subsets of $\mathbb{R}$ verify (1)-(4) of Proposition 1, if $u$ and $v$ are defined by

$$
\begin{gathered}
u(x)=\left\{\begin{array}{ll}
0, & \text { if } x \notin M_{0} \\
\sup \left\{\alpha \in[0,1]: x \in M_{\alpha}\right\}, & \text { if } x \in M_{0}
\end{array},\right. \\
v(x)=\left\{\begin{array}{ll}
1, & \text { if } x \notin M^{0} \\
1-\sup \left\{\alpha \in[0,1]: x \in M^{\alpha}\right\}, & \text { if } x \in M^{0}
\end{array},\right.
\end{gathered}
$$

then $\langle u, v\rangle \in \mathbb{I F}^{1}$.

Lemma 2.7. [3] Let I be a dense subset in $[0,1]$. If $[\langle u, v\rangle]_{\alpha}=[\langle w, z\rangle]_{\alpha}$ and $[\langle u, v\rangle]^{\alpha}=[\langle w, z\rangle]^{\alpha}$, $\forall \alpha \in I$, then $\langle u, v\rangle=\langle w, z\rangle$.

Definition 2.8. [7] Let $\left\langle u_{1}, v_{1}\right\rangle,\left\langle u_{2}, v_{2}\right\rangle \in \mathbb{I F}^{1}$. If there exists $\langle w, z\rangle \in \mathbb{I F}^{1}$ such that $\left\langle u_{1}, v_{1}\right\rangle=$ $\left\langle u_{2}, v_{2}\right\rangle+\langle w, z\rangle$, then $\langle w, z\rangle$ is called Hukuhara difference of $\left\langle u_{1}, v_{1}\right\rangle$ and $\left\langle u_{2}, v_{2}\right\rangle$ denoted by $\left\langle u_{1}, v_{1}\right\rangle \ominus\left\langle u_{2}, v_{2}\right\rangle$.

The concept of intuitionistic fuzzy Hukuhra difference is introduced by the autors in [3].

Definition 2.9. [7, 18] The generalized Hukuhara difference of two intuitionistic fuzzy numbers $\left\langle u_{1}, v_{1}\right\rangle,\left\langle u_{2}, v_{2}\right\rangle \in \mathbb{I F}^{1}$ is as follows:

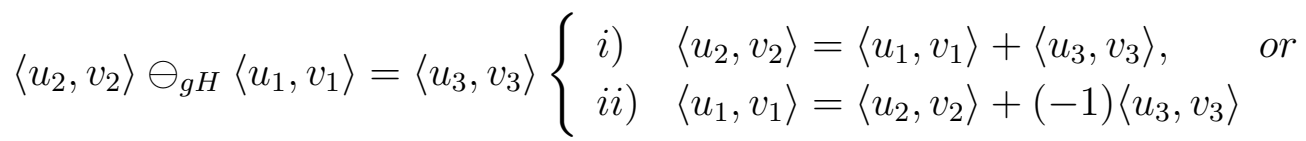

Definition 2.10. [2] Let $f:[a, b] \rightarrow \mathbb{I F}^{1}$ and $t_{0} \in[a, b]$. We say that $f$ is generalized Hukuhara differentiable at $t_{0}$ if there exists $f^{\prime}\left(t_{0}\right) \in \mathbb{I F}^{1}$ such that:

$$
f^{\prime}\left(t_{0}\right)=\lim _{h \rightarrow 0^{+}} \frac{f\left(t_{0}+h\right) \ominus_{g H} f\left(t_{0}\right)}{h}=\lim _{h \rightarrow 0^{-}} \frac{f\left(t_{0}\right) \ominus_{g H} f\left(t_{0}-h\right)}{h} .
$$


Definition 2.11. [19] $F:[a, b] \longrightarrow \mathbb{I F}^{1}$ is strongly measurable if $\forall \alpha \in[0,1]$, the set-valued mappings $F_{\alpha}:[a, b] \longrightarrow \mathcal{P}_{K}(\mathbb{R})$ defined by $F_{\alpha}(t)=[F(t)]^{\alpha}$ and $F^{\alpha}:[a, b] \longrightarrow \mathcal{P}_{K}(\mathbb{R})$ defined by $F^{\alpha}(t)=[F(t)]_{\alpha}$ are Lebesgue measurable.

Definition 2.12. $[18,19]$ Let $F:[a, b] \longrightarrow \mathbb{I F}^{1}$. We say that $F$ is integrable on $[a, b]$ if there exists $\langle u, v\rangle \in \mathbb{I F}^{1}$ such that for each $\alpha \in[0,1]$ :

$$
\begin{aligned}
{\left[\int_{a}^{b} F(t) d t\right]^{\alpha} } & =\left\{\int_{a}^{b} f(t) d t \mid f:[a, b] \longrightarrow \mathbb{R} \text { is a measurable selection for } F_{\alpha}\right\} \\
{\left[\int_{a}^{b} F(t) d t\right]_{\alpha} } & =\left\{\int_{a}^{b} f(t) d t \mid f:[a, b] \longrightarrow \mathbb{R} \text { is a measurable selection for } F_{\alpha}\right\} \\
{[\langle u, v\rangle]^{\alpha} } & =\left[\int_{a}^{b} F(t) d t\right]^{\alpha}, \\
{[\langle u, v\rangle]_{\alpha} } & =\left[\int_{a}^{b} F(t) d t\right]_{\alpha},
\end{aligned}
$$

and we write $\int_{a}^{b} F(t) d t=\langle u, v\rangle$.

Let $d_{\infty}: \mathbb{I} \mathbb{F}^{1} \times \mathbb{I} \mathbb{F}^{1} \longrightarrow[0,+\infty[$ be a mapping defined by:

$$
\begin{aligned}
d_{\infty}(\langle u, v\rangle,\langle w, z\rangle)= & \frac{1}{4} \sup _{0 \leq \alpha \leq 1}\left|[\langle u, v\rangle]_{r}^{+}(\alpha)-[\langle w, z\rangle]_{r}^{+}(\alpha)\right| \\
& +\frac{1}{4} \sup _{0 \leq \alpha \leq 1}\left|[\langle u, v\rangle]_{l}^{+}(\alpha)-[\langle w, z\rangle]_{l}^{+}(\alpha)\right| \\
& +\frac{1}{4} \sup _{0 \leq \alpha \leq 1}\left|[\langle u, v\rangle]_{r}^{-}(\alpha)-[\langle w, z\rangle]_{r}^{-}(\alpha)\right| \\
& +\frac{1}{4} \sup _{0 \leq \alpha \leq 1}\left|[\langle u, v\rangle]_{l}^{-}(\alpha)-[\langle w, z\rangle]_{l}^{-}(\alpha)\right|
\end{aligned}
$$

Then we have the following result.

Proposition 2.13. [3] $\left(\mathbb{I F} \mathbb{F}^{1}, d_{\infty}\right)$ is a complet metric space.

\subsection{Fractional integral and fractional derivatives of an intuitionistic fuzzy function}

Definition 2.14. [10] Let $F(t) \in L\left([0, T], \mathbb{I F}^{1}\right)$. The intuitionistic fuzzy fractional integral of order $q \in[0,1]$ of $F$ denoted by

$$
I^{q} F(t):=\frac{1}{\Gamma(q)} \int_{0}^{t}(t-s)^{q-1} F(s) d s
$$

is defined by

$$
\begin{aligned}
& {\left[I^{q} F(t)\right]^{\alpha}=\left[I^{\alpha} F_{l}^{-}(t ; \alpha), I^{q} F_{r}^{-}(t ; \alpha)\right],} \\
& {\left[I^{q} F(t)\right]_{\alpha}=\left[I^{\alpha} F_{l}^{+}(t ; \alpha), I^{q} F_{r}^{+}(t ; \alpha)\right],}
\end{aligned}
$$

where $\Gamma($.$) is the Euler gamma function.$ 
Proposition 2.15. [10] Let $F, G \in L\left([0, T], \mathbb{I F}^{1}\right)$ and $a \in \mathbb{I F}^{1}$, then we have

1. $I^{q}(a F)(t)=a I^{q} F(t)$.

2. $I^{q}(F+G)(t)=I^{q} F(t)+I^{q} G(t)$.

3. $I^{q_{1}} I^{q_{2}} F(t)=I^{q_{1}+q_{2}} F(t)$,where $\left(q_{1}, q_{2}\right) \in[0,1]^{2}$.

Definition 2.16. [10] Let $F \in C\left([0, T], \mathbb{I F}^{1}\right) \cap L\left([0, T], \mathbb{I F}^{1}\right)$. The function $F$ is called intuitionistic fuzzy Caputo fractional differentiable of order $0<q<1$ at $x$ if there exists an element ${ }^{c} D^{q} F(x) \in \mathbb{I F}^{1}$ such that

$$
{ }^{c} D^{q} F(t)=\frac{1}{\Gamma(q)} \int_{0}^{t}(t-s)^{q-1} F^{\prime}(s) d s .
$$

Example 2.17. Consider the intuitionistic fuzzy function $\langle u, v\rangle(t)=t C$, where $C=\left(a_{1} ; a_{2} ; a_{3} ; a_{4} ; a_{1}^{\prime} ; a_{2} ; a_{3} ; a_{4}^{\prime}\right)$ is a trapezoidal intuitionistic fuzzy number. In this example we calculate the intuitionistic fuzzy Caputo fractional derivative of the function $\langle u, v\rangle(t)$. For this purpose, we start by giving the $g H$-derivative of $\langle u, v\rangle(t)$ as follows:

$$
\langle u, v\rangle^{\prime}(t)=\lim _{h \rightarrow 0} \frac{\langle u, v\rangle(t+h) \ominus_{g H}\langle u, v\rangle(t)}{h}=\lim _{h \rightarrow 0} \frac{(t+h) C \ominus_{g H} t C}{h}=C .
$$

This implies that

$$
\langle u, v\rangle^{\prime}(t)=C .
$$

Since $[C]^{\alpha}=\left[a_{2}-\alpha\left(a_{2}-a_{1}^{\prime}\right), a_{3}+\alpha\left(a_{4}-a_{3}\right)\right]$ and $[C]_{\alpha}=\left[a_{1}+\alpha\left(a_{2}-a_{1}\right), a_{4}-\alpha\left(a_{4}^{\prime}-a_{3}\right)\right]$ we have

$$
\begin{gathered}
{\left[{ }^{c} D^{q}\langle u, v\rangle(x)\right]^{\alpha}=\left[I^{1-q}\left(\langle u, v\rangle^{\prime}(t)\right)\right]^{\alpha}=\left[I^{1-q} C\right]^{\alpha}} \\
{\left[{ }^{c} D^{q}\langle u, v\rangle(x)\right]_{\alpha}=\left[I^{1-q}\left(\langle u, v\rangle^{\prime}(t)\right)\right]^{\alpha}=\left[I^{1-q} C\right]_{\alpha}} \\
{\left[I^{1-q} C\right]^{\alpha}=\frac{1}{\Gamma(q)} \int_{0}^{t}(t-s)^{q-1}\left[a_{2}-\alpha\left(a_{2}-a_{1}^{\prime}\right), a_{3}+\alpha\left(a_{4}-a_{3}\right)\right] d s} \\
{\left[I^{1-q} C\right]_{\alpha}=\frac{1}{\Gamma(q)} \int_{0}^{t}(t-s)^{q-1}\left[a_{1}+\alpha\left(a_{2}-a_{1}\right), a_{4}-\alpha\left(a_{4}^{\prime}-a_{3}\right)\right] d s} \\
{\left[I^{1-q} C\right]^{\alpha}=\frac{t^{q}}{\Gamma(q) \times q}\left[a_{2}-\alpha\left(a_{2}-a_{1}^{\prime}\right), a_{3}+\alpha\left(a_{4}-a_{3}\right)\right]} \\
{\left[I^{1-q} C\right]_{\alpha}=\frac{t^{q}}{\Gamma(q) \times q}\left[a_{1}+\alpha\left(a_{2}-a_{1}\right), a_{4}-\alpha\left(a_{4}^{\prime}-a_{3}\right)\right]} \\
{\left[I^{1-q} C\right]^{\alpha}=\frac{t^{q}}{\Gamma(q+1)}[C]^{\alpha}} \\
{\left[I^{1-q} C\right]_{\alpha}=\frac{t^{q}}{\Gamma(q+1)}[C]_{\alpha} .}
\end{gathered}
$$

Thus

$$
\begin{aligned}
{ }^{c} D^{q}\langle u, v\rangle(t) & =\frac{t^{q}}{\Gamma(q+1)} C \\
{ }^{c} D^{q}\langle u, v\rangle(t) & =\frac{t^{q-1}}{\Gamma(q+1)}\langle u, v\rangle(t) .
\end{aligned}
$$




\subsection{Laplace transform of Caputo fractional derivative}

In order to establish the Laplace transform of Caputo fractional derivative [12], we write the Caputo derivative under the form

$$
{ }^{c} D^{\alpha} f(t):=I^{n-\alpha} f^{(n)}(t) .
$$

By using the formula of Laplace transform of Riemann-Liouville fractional integral, we have [1]

$$
L\left\{{ }^{c} D^{\alpha} f(t), s\right\}:=L\left\{I^{n-\alpha} f^{(n)}(t), s\right\}=s^{\alpha-n} G(s) .
$$

Were $G(s)$ is given by

$$
G(s)=s^{n} F(s)-\sum_{k=0}^{n-1} s^{n-k-1} f^{(k)}(0)=s^{n} F(s)-\sum_{k=0}^{n-1} s^{k} f^{(n-k-1)}(0) .
$$

Finaly, the Laplace transform of Caputo fractional derivative is,

$$
L\left\{{ }^{c} D^{\alpha} f(t), s\right\}=s^{\alpha} F(s)-\sum_{k=0}^{n-1} s^{\alpha-k-1} f^{(k)}(0) .
$$

Remark 2.18. [12] For $0<\alpha<1$ we have

$$
L\left\{{ }^{c} D^{\alpha} f(t), s\right\}=s^{\alpha} F(s)-f(0) .
$$

\subsection{Laplace transform of Mittag-Leffler function}

The Mittag-Leffler function is an important function that finds widespread use in the world of fractional calculus. Just as the exponential naturally arises out of the solution to integer order differential equations, the Mittag-Leffler function plays an analogous role in the solution of noninteger order differential equations. In fact, the exponential function itself is a very special form, one of an infinite set of the seemingly ubiquitous functions.

Definition 2.19. [1] We recall that the Mittag-Leffler function is given by

$$
E_{\alpha}(z):=\sum_{k=0}^{+\infty} \frac{z^{k}}{\Gamma(\alpha k+1)}, \quad \alpha \in \mathbb{C}, R(\alpha)>0, z \in \mathbb{C} .
$$

The general form is given by

$$
E_{\alpha, \beta}(z):=\sum_{k=0}^{+\infty} \frac{z^{k}}{\Gamma(\alpha k+\beta)}, \quad \beta, \alpha \in \mathbb{C}, R(\beta)>0, R(\alpha)>0, z \in \mathbb{C} .
$$

Then we have

$$
L\left\{E_{\alpha}\left(\lambda t^{\alpha}\right) ; s\right\}=\frac{s^{\alpha-1}}{s^{\alpha}-\lambda}, s>\lambda^{\frac{1}{\alpha}} .
$$


Indeed, for $s>\lambda$, using the series expansion of the exponential function we have

$$
\begin{aligned}
\frac{1}{s-\lambda} & =\int_{0}^{+\infty} e^{-s t} e^{\lambda t} d t \\
& =\sum_{k=0}^{\infty} \frac{\lambda^{k}}{k !} \int_{0}^{+\infty} e^{-s t} t^{k} d t \\
& =\sum_{k=0}^{\infty} \frac{\lambda^{k}}{k !} \frac{k !}{s^{k+1}} \\
& =\sum_{k=0}^{\infty} \frac{\lambda^{k}}{s^{k+1}}
\end{aligned}
$$

Then similarly for the Mittag-Leffler function we obtain

$$
\begin{aligned}
& L\left\{E_{\alpha}\left(\lambda t^{\alpha}\right) ; s\right\}=\sum_{k=0}^{\infty} \frac{\lambda^{k}}{\Gamma(\alpha k+1)} \int_{0}^{+\infty} e^{-s t} t^{\alpha k} d t \\
& L\left\{E_{\alpha}\left(\lambda t^{\alpha}\right) ; s\right\}=\sum_{k=0}^{\infty} \frac{\lambda^{k}}{\Gamma(\alpha k+1)} \frac{\Gamma(\alpha k+1)}{s^{\alpha k+1}} \\
& L\left\{E_{\alpha}\left(\lambda t^{\alpha}\right) ; s\right\}=\sum_{k=0}^{\infty} \frac{\lambda^{k}}{s^{\alpha k+1}}=s^{\alpha-1} \sum_{k=0}^{\infty} \frac{\lambda^{k}}{\left(s^{\alpha}\right)^{k+1}} \\
& L\left\{E_{\alpha}\left(\lambda t^{\alpha}\right) ; s\right\}==\frac{s^{\alpha-1}}{s^{\alpha}-\lambda}, s>\lambda^{\frac{1}{\alpha}} .
\end{aligned}
$$

The Laplace transform of Mittag-Leffler function in two parameters is given by

$$
L\left\{t^{\beta-1} E_{\alpha, \beta}\left(-\lambda t^{\alpha}\right) ; s\right\}=\frac{s^{\alpha}-\beta}{s^{\alpha}+\lambda}, R(s)>|\lambda|^{\frac{1}{\alpha}}
$$

\section{Existence and uniqueness resuts}

In this section we study the existence and uniqueness of the solution for the following initial value problem.

$$
\left\{\begin{array}{l}
{ }^{c} D^{\alpha} x(t)=f(t, x(t)) ; \quad t \in\left[t_{0}, T\right] \\
x\left(t_{0}\right)=x_{0} \in \mathbb{I} \mathbb{F}
\end{array}\right.
$$

where ${ }^{c} D^{q}$ is the Caputo derivative of $x(t)$ at order $\left.\alpha \in\right] 0,1[$.

For this purpose, we need some notations and definitions:

- $C\left(\left[t_{0}, T\right], \mathbb{I} \mathbb{F}^{1}\right)$ denotes the space of all continuous functions from $\left[t_{0}, T\right]$ to $\mathbb{I F}^{1}$.

- $B_{r}\left(x_{0}, r\right)=\left\{y \in \mathbb{I F}^{1}, d_{\infty}\left(x_{0}, y\right) \leq r\right\}$ denotes the closed ball of $\mathbb{I F}^{1}$.

Definition 3.1. A function $x:\left[t_{0}, T\right] \rightarrow \mathbb{I} \mathbb{F}^{1}$ is said to be a solution of (3) if $x$ is continuous such that $x\left(t_{0}\right)=x_{0}$ and ${ }^{c} D^{\alpha} x(t)=f(t, x(t)), t \in\left[t_{0}, T\right]$. 
Definition 3.2. [11] An intuitionistic fuzzy function $x:\left[t_{0}, T\right] \rightarrow \mathbb{I F}^{1}$ is called $d$-increasing (d-decreasing) on $\left[t_{0}, T\right]$ if for every $r \in\left[t_{0}, T\right]$ the real function $t \rightarrow d\left([x(t)]^{r} \cup[x(t)]_{r}\right)$ is non-decreasing (non-increasing), respectively.

Remark 3.3. If $x:\left[t_{0}, T\right] \rightarrow \mathbb{I F}^{1}$ is $d$-increasing or $d$-decreasing on $\left[t_{0}, T\right]$, then we say that $x(t)$ is $d$-monotone on $\left[t_{0}, T\right]$.

Lemma 3.4. [11] Let $f:\left[t_{0}, T\right] \times \mathbb{I F}^{1} \rightarrow \mathbb{I F}^{1}$ such that $f$ is continuous with respect to the first variable $t$ on $\left[t_{0}, T\right]$. A d-monotone intuitionistic fuzzy function $x(t) \in C\left(\left[t_{0}, T\right], \mathbb{I F}^{1}\right)$ is a solution of initial value problem (3) if and only if

1) $x$ satisfies the integral equation $x(t) \ominus_{g H} x_{0}=\frac{1}{\Gamma(\alpha)} \int_{t_{0}}^{t}(t-s)^{\alpha-1} f(s, x(s)) d s$.

2) The function $t \rightarrow I^{q} f(t, x(t))$ is d-increasing on $\left[t_{0}, T\right]$.

Proof. [11] The proof of this lemma is similar to the proof of Theorem 3 in [11].

Theorem 3.5. $[9,24]$ Let $C$ be a non-empty closed subset of a Banach space $X$ and let $\left(k_{n}\right)_{n \geq 0}$ be a sequence such that $\sum_{n \geqslant 0}^{\infty} k_{n}$ converges. Moreover, let the operator $T: C \rightarrow C$ satisfy the following inequality

$$
\left\|T^{n} x-T^{n} y\right\| \leq k_{n}\|x-y\| \quad \forall n \in \mathbb{N} \quad \text { and } \quad \forall x, y \in C .
$$

Then the operator $T$ has a uniquely defined fixed point $x^{*}$. In addition, the sequence $\left\{T^{n} x_{0}\right\}_{n \geq 0}$ converges to $x^{*}$ for every $x_{0} \in C$.

\subsection{Fundamental theorem}

Theorem 3.6. Let $f:\left[t_{0}, T\right] \times \mathbb{I F}^{1} \rightarrow \mathbb{I F}^{1}$ be a bounded and continuous function on $\left[t_{0}, T\right]$ such that:

$\left.H_{1}\right)$ There exists a positive constant $M$ such that

$$
d_{\infty}\left(f(t, x), 0_{\mathbb{I F}^{1}}\right)<M \quad \forall(t, x) \in\left[t_{0}, T\right] \times C\left(\left[t_{0}, T\right], \mathbb{I F}^{1}\right) .
$$

$\left.H_{2}\right)$ There exists a continuous function $k:\left[t_{0}, T\right] \rightarrow \mathbb{R}^{+}$such that,

$$
d_{\infty}\left(f(t, x), f(t, y)<k(t) d_{\infty}(x, y) \quad(x, y) \in C\left(\left[t_{0}, T\right], \mathbb{I F}^{1}\right) \times C\left(\left[t_{0}, T\right], \mathbb{I F}^{1}\right) \text { and } t \in\left[t_{0}, T\right] .\right.
$$

Then the problem (3) has a unique solution.

Proof. The proof of this theorem will be given in two steps.

Step 1. (Existence of a solution of the problem (3). To show that the problem (3) has at least one solution defined on $\left[t_{0}, T\right]$ we use the Schauder fixed point theorem, [22]. 
Let $\mathbf{T}: C\left(\left[t_{0}, T\right], \mathbb{I F}^{1}\right) \rightarrow C\left(\left[t_{0}, T\right], \mathbb{I F}^{1}\right.$ be the operator defined as follows:

$$
\mathbf{T} x(t) \ominus_{g H} x_{0}=\frac{1}{\Gamma(\alpha)} \int_{t_{0}}^{t}(t-s)^{\alpha-1} f(t, x(t)) d s .
$$

Let $x \in C\left(\left[t_{0}, T\right], \mathbb{I F}^{1}\right)$ then $\mathbf{T} x$ is continuous and one has

$$
\begin{aligned}
& d_{\infty}\left(\mathbf{T} x(t), 0_{\mathbb{I} \mathbb{F}^{1}}\right) \leq d_{\infty}\left(x_{0}, 0_{\mathbb{I} \mathbb{F}^{1}}\right)+\frac{1}{\Gamma(\alpha)} \int_{t_{0}}^{t}(t-s)^{\alpha-1} d_{\infty}\left(f(t, x(t)), 0_{\mathbb{\mathbb { F } ^ { 1 }}}\right) d s, \\
& d_{\infty}\left(\mathbf{T} x(t), 0_{\mathbb{I} \mathbb{F}^{1}}\right) \leq d_{\infty}\left(x_{0}, 0_{\mathbb{\mathbb { F } ^ { 1 }}}\right)+\frac{M\left(T-t_{0}\right)^{\alpha}}{\Gamma(\alpha+1)}:=\rho .
\end{aligned}
$$

Hence, $\mathbf{T} x \in C\left(\left[t_{0}, T\right], \mathbb{I F}^{1}\right)$, it follows that $\mathbf{T}$ transforms the ball

$$
B_{\rho}\left(0_{\mathbb{I F}}, \rho\right)=\left\{y \in C\left(\left[t_{0}, T\right], \mathbb{I F}^{1}\right) d_{\infty}\left(0_{\mathbb{I F}}, y\right) \leq \rho\right\}
$$

into itself.

Let us show that $\mathbf{T}$ is continuous.

Let $\left(x_{n}\right)_{n} \subset B_{\rho}$ such that $x_{n}$ converges to $x$ in $B_{\rho}$, and $t \in\left[t_{0}, T\right]$ we have

$$
\begin{aligned}
d_{\infty}\left(\mathbf{T} x_{n}(t), \mathbf{T} x(t)\right) & \left.=d_{\infty}\left(\mathbf{T} x_{n}(t) \ominus_{g H} x_{0}, \mathbf{T} x\right)(t) \ominus_{g H} x_{0}\right) \\
& \leq \frac{1}{\Gamma(\alpha+1)} \int_{t_{0}}^{t}(t-s)^{\alpha-1} d_{\infty}\left(f\left(t, x_{n}(t)\right), f(t, x(t))\right) d s
\end{aligned}
$$

Since $f$ is continuous and satisfies $H_{2}$, then we have

$$
d_{\infty}\left(\mathbf{T} x_{n}(t), \mathbf{T} x(t)\right) \leq \frac{1}{\Gamma(\alpha+1)} \int_{t_{0}}^{t}(t-s)^{\alpha-1} k(s) d_{\infty}\left(x_{n}(s), x(s)\right) d s .
$$

By going to the limit and using Lebesgue's dominated convergence theorem, we obtain

$$
\lim _{n \rightarrow \infty} d_{\infty}\left(\mathbf{T} x_{n}(t), \mathbf{T} x(t)\right)=0 .
$$

Let us also show that $\mathbf{T} B_{\rho}$ is bounded and equicontinuous on $\left[t_{0}, T\right]$.

We have $\mathbf{T} B_{\rho} \subset B_{\rho}$, then $\mathbf{T} B_{\rho}$ is bounded.

Let $x \in B_{\rho}$ and $t_{1}, t_{2} \in\left[t_{0}, T\right]$ such that $t_{1}<t_{2}$, then we have

$$
d_{\infty}\left(\mathbf{T} x\left(t_{1}\right), \mathbf{T} x\left(t_{2}\right)\right) \leq \frac{1}{\Gamma(\alpha)} d_{\infty}\left(\int_{t_{0}}^{t_{1}}\left(t_{1}-s\right)^{\alpha-1} f(s, x(s)) d s, \int_{t_{0}}^{t_{2}}\left(t_{2}-s\right)^{\alpha-1} f(s, x(s)) d s\right) .
$$

Since

$\int_{t_{0}}^{t_{2}}\left(t_{2}-s\right)^{\alpha-1} f(s, x(s)) d s=\int_{t_{0}}^{t_{1}}\left(t_{2}-s\right)^{\alpha-1} f(s, x(s)) d s+\int_{t_{1}}^{t_{2}}\left(t_{2}-s\right)^{\alpha-1} f(s, x(s)) d s$,

then

$$
\begin{gathered}
d_{\infty}\left(\left(\mathbf{T} x\left(t_{1}\right), \mathbf{T} x\left(t_{2}\right)\right) \leq \frac{1}{\Gamma(\alpha)} \int_{t_{0}}^{t_{1}}\left|\left(t_{1}-s\right)^{\alpha-1}-\left(t_{1}-s\right)^{\alpha-1}\right| d_{\infty}\left(f(s, x(s)) d s, 0_{\mathbb{I F}^{1}}\right)\right. \\
+\int_{t_{1}}^{t_{2}}\left(t_{2}-s\right)^{\alpha-1} d_{\infty}\left(f(s, x(s)) d s, 0_{\mathbb{\mathbb { F } ^ { 1 }}}\right) d s .
\end{gathered}
$$


It follows that

$$
d_{\infty}\left(\mathbf{T} x\left(t_{1}\right), \mathbf{T} x\left(t_{2}\right)\right) \leq \frac{M}{\Gamma(\alpha+1)}\left(\left(t_{2}-t_{1}\right)^{\alpha}+\left(t_{2}^{\alpha}-t_{1}^{\alpha}\right)\right) .
$$

Hence,

$$
\lim _{t_{1} \rightarrow t_{2}} d_{\infty}\left(\mathbf{T} x\left(t_{1}\right), \mathbf{T} x\left(t_{2}\right)\right)=0,
$$

which shows that $\mathbf{T} B_{\rho}$ is equicontinuous, by using Arzelà-Ascoli theorem [23] we deduce that $\mathbf{T} B_{\rho}$ is relatively compact.

Finally, by using the Schauder fixed point theorem, we can conclude that the operator $\mathbf{T}$ has a fixed point $x(t)$, which is a solution of the problem (3).

Step 2. (Uniqueness of the solution) To show the uniqueness of the solution, we suppose that there exists another solution $y(t):\left[t_{0}, T\right] \rightarrow C\left(\left[t_{0}, T\right], \mathbb{I F}^{1}\right)$ for the system (3), then we have

$$
\begin{gathered}
d_{\infty}(\mathbf{T} x(t), \mathbf{T} y(t))=d_{\infty}\left(\mathbf{T} x(t) \ominus_{g H} x_{0}, \mathbf{T} y(t) \ominus_{g H} x_{0}\right) \\
\leq \frac{1}{\Gamma(\alpha)} \int_{t_{0}}^{t}(t-s)^{\alpha-1} d_{\infty}(f(t, x(t)), f(t, y(t))) d s \\
\leq \frac{1}{\Gamma(\alpha)} \int_{t_{0}}^{t}(t-s)^{\alpha-1} k(t) d_{\infty}(x, y) d s .
\end{gathered}
$$

Taking $K=\sup _{t \in\left[t_{0}, T\right]} k(t)$, we obtain

$$
d_{\infty}(\mathbf{T} x(t), \mathbf{T} y(t)) \leq \frac{K}{\Gamma(\alpha)} \int_{t_{0}}^{t}(t-s)^{\alpha-1} d_{\infty}(x(s), y(s)) d s
$$

We take the sup on both sides of the previous inequality we will have,

$$
\sup _{t \in\left[t_{0}, T\right]} d_{\infty}(\mathbf{T} x(t),(\mathbf{T} y)(t)) \leq \frac{K\left(t-t_{0}\right)^{\alpha}}{\Gamma(\alpha+1)} \sup _{t \in\left[t_{0}, T\right]} d_{\infty}(x(t), y(t)) .
$$

By the induction method one can conclude that for every $n \in \mathbb{N}^{*}$ and for every $x, y \in C\left(\left[t_{0}, T\right], \mathbb{I F}^{1}\right)$ :

$$
\sup _{t \in\left[t_{0}, T\right]} d_{\infty}\left(\mathbf{T}^{n} x(t), \mathbf{T}^{n} y(t)\right) \leq \frac{K^{n}\left(t-t_{0}\right)^{n \alpha}}{\Gamma(n \alpha+1)} \sup _{t \in\left[t_{0}, T\right]} d_{\infty}(x(t), y(t)),
$$

where $\mathbf{T}^{n}: C\left(\left[t_{0}, T\right], \mathbb{I F}^{1}\right) \rightarrow C\left(\left[t_{0}, T\right], \mathbb{I F}^{1}\right)$ is defined by

$$
\mathbf{T}^{n} x(t) \ominus_{g H} x_{0}=\frac{1}{\Gamma(\alpha)} \int_{t_{0}}^{t}(t-s)^{\alpha-1} f\left(t,\left(\mathbf{T}^{n-1} x\right) x(t)\right) d s .
$$

Now we shall prove that for every $n \in \mathbb{N}^{*}$ we have

$$
\sup _{t \in\left[t_{0}, T\right]} d_{\infty}\left(\mathbf{T}^{n} x(t), \mathbf{T}^{n} y(t)\right) \leq \frac{K^{n}\left(t-t_{0}\right)^{n \alpha}}{\Gamma(n \alpha+1)} \sup _{t \in\left[t_{0}, T\right]} d_{\infty}(x(t), y(t)) .
$$

- For $n=1$ the statement (4) is trivially true. 
- We suppose that

$$
\sup _{t \in\left[t_{0}, T\right]} d_{\infty}\left(\mathbf{T}^{n} x(t), \mathbf{T}^{n} y(t)\right) \leq \frac{K^{n}\left(t-t_{0}\right)^{n \alpha}}{\Gamma(n \alpha+1)} \sup _{t \in\left[t_{0}, T\right]} d_{\infty}(x(t), y(t))
$$

and let us show that

$$
\sup _{t \in\left[t_{0}, T\right]} d_{\infty}\left(\left(\mathbf{T}^{n+1} x\right)(t),\left(\mathbf{T}^{n+1} y\right)(t)\right) \leq \frac{K^{n+1}\left(t-t_{0}\right)^{(n+1) \alpha}}{\Gamma((n+1) \alpha+1)} \sup _{t \in\left[t_{0}, T\right]} d_{\infty}(x(t), y(t)) .
$$

We have

$$
\begin{aligned}
d_{\infty}\left(\mathbf{T}^{n+1} x(t), \mathbf{T}^{n+1} y(t)\right) & =d_{\infty}\left(\mathbf{T}^{n+1} x(t) \ominus_{g H} x_{0}, \mathbf{T}^{n+1} y(t) \ominus_{g H} x_{0}\right) \\
d_{\infty}\left(\mathbf{T}^{n+1} x(t), \mathbf{T}^{n+1} y(t)\right) & =d_{\infty}\left(\mathbf{T T}^{n} x(t) \ominus_{g H} x_{0}, \mathbf{T T}^{n} y(t) \ominus_{g H} x_{0}\right) \\
& \leq \frac{K}{\Gamma(\alpha)} \int_{t_{0}}^{t}(t-s)^{\alpha-1} d_{\infty}\left(\mathbf{T}^{n} x(s), \mathbf{T}^{n} y(s)\right) d s .
\end{aligned}
$$

Taking the sup on $t$ and $s$, we obtain

$$
\begin{gathered}
\sup _{t \in\left[t_{0}, T\right]} d_{\infty}\left(\mathbf{T}^{n+1} x(t), \mathbf{T}^{n+1} y(t)\right) \leq \frac{K}{\Gamma(\alpha)} \int_{t_{0}}^{t}(t-s)^{\alpha-1} \sup _{s \in\left[t_{0}, T\right]} d_{\infty}\left(\mathbf{T}^{n} x(s), \mathbf{T}^{n} y(s)\right) d s \\
\sup _{t \in\left[t_{0}, T\right]} d_{\infty}\left(\mathbf{T}^{n+1} x(t), \mathbf{T}^{n+1} y(t)\right) \leq \frac{K}{\Gamma(\alpha)} \int_{t_{0}}^{t}(t-s)^{\alpha-1} \times \frac{K^{n}\left(s-t_{0}\right)^{n \alpha}}{\Gamma(n \alpha+1)} \sup _{s \in\left[t_{0}, T\right]} d_{\infty}(x(s), y(s)) d s \\
\sup _{t \in\left[t_{0}, T\right]} d_{\infty}\left(\mathbf{T}^{n+1} x(t), \mathbf{T}^{n+1} y(t)\right) \leq \frac{K^{n+1}}{\Gamma(\alpha) \Gamma(n \alpha+1)} \int_{t_{0}}^{t}(t-s)^{\alpha-1} \times\left(s-t_{0}\right)^{n \alpha} \sup _{s \in\left[t_{0}, T\right]} d_{\infty}(x(s), y(s)) d s .
\end{gathered}
$$

Since the Beta function [12] is defined by

$$
B(p, q)=\int_{0}^{1} s^{p-1} \times(1-s)^{q-1}=\frac{\Gamma(p) \Gamma(q)}{\Gamma(p+q)}, \quad p, q>0 .
$$

It follows that

$$
\begin{gathered}
\sup _{t \in\left[t_{0}, T\right]} d_{\infty}\left(\mathbf{T}^{n+1} x(t), \mathbf{T}^{n+1} y(t)\right) \leq \frac{K^{n+1}}{\Gamma(\alpha) \Gamma(n \alpha+1)} \times\left(t-t_{0}\right)^{(n+1) \alpha} \frac{\Gamma(\alpha) \Gamma(n \alpha+1)}{\Gamma(\alpha+n \alpha+1)} \sup _{t \in\left[t_{0}, T\right]} d_{\infty}(x(t), y(t)) \\
\sup _{t \in\left[t_{0}, T\right]} d_{\infty}\left(\mathbf{T}^{n+1} x(t), \mathbf{T}^{n+1} y(t)\right) \leq \frac{K^{n+1}(t-a)^{(n+1)}}{\Gamma((1+n) \alpha+1)} \sup _{t \in\left[t_{0}, T\right]} d_{\infty}(x(t), y(t)) .
\end{gathered}
$$

Therefore, the statement (4) is true for all $n \in \mathbb{N}^{*}$.

We observe (see [12]) that the series $\sum_{n \geqslant 0}^{\infty} \frac{\left(K\left(t-t_{0}\right)\right)^{n}}{\Gamma(n \alpha+1)}$ converges to the Mittag-Leffler function $E_{\alpha, 1}\left(K\left(t-t_{0}\right)\right)$, then by the Theorem 3.5 we can deduce that the operator $\mathbf{T}$ has a unique fixed point $x$, which is the solution of our problem (3). 


\section{Stability of the solution}

Our aim in this section is to study the stability of the solution for the following nonlinear fractional differential equation. For this purpose, we extend the Lyapunov direct method to introduce the stability in the Mittag-Leffler sense of the trivial solution $\left(x=0_{\mathbb{I F}}\right)$ for the following nonlinear system:

$$
\left\{\begin{array}{l}
{ }^{c} D^{\alpha} x(t)=f(t, x(t)) \quad ; t \in[0,+\infty[ \\
x(0)=x_{0}
\end{array},\right.
$$

where $f:\left[0,+\infty\left[\times \mathbb{I F}^{1} \rightarrow \mathbb{I F}^{1}\right.\right.$ is an intuitionistic fuzzy continuous function in $t$ locally Lipschitz in $x$ such that $f\left(t, 0_{\mathbb{I F}}\right)=0_{\mathbb{I F}}$.

The existence and uniqueness results are discussed in Section 3 for the case $t \in\left[t_{0}, T\right]$. Since the fact $f\left(t, 0_{\mathbb{I F}}\right)=0_{\mathbb{I F}}$ means that the intuitionistic fuzzy zero function is a solution of initial value problem (5), then our purpose in this work is to study the stability of the trivial solution of the problem (5).

Definition 4.1. [15] The intuitionistic fuzzy number $x_{e}$ is an equilibrium point of the system (5) if and only if $f\left(t, x_{e}\right)=0_{\mathbb{I F}}$.

Remark 4.2. For convenience, we state all definitions and theorems for the case when the equilibrium point is the origin $x_{e}=0_{\mathbb{I F}}$.

There is no loss of generality in doing so because any equilibrium point can be shifted to the origin via a change of variables. Suppose the intuitionistic fuzzy equilibrium point for (5) is $x_{e} \neq 0_{\mathbb{I F}}$ and consider the change of variable $X=x \ominus_{g H} x_{e}$. The fractional derivative of $X(t)$ is given by

$$
{ }^{c} D^{\alpha} X(t)={ }^{c} D^{\alpha}\left(x(t) \ominus_{g H} x_{e}\right)=f\left(t, X(t)+x_{e}\right)=F(t, X(t)),
$$

where $F\left(t, 0_{\mathbb{I F}}\right)=0_{\mathbb{I F}}$ and the system has equilibrium at the origin.

Definition 4.3. The trivial solution of (5) is said to be:

(1) stable, iff for any $\epsilon>0$, there exists $\delta>0$, such that

$$
d_{\infty}\left(x_{0}, 0_{\mathbb{I F}}\right)<\delta \Rightarrow d_{\infty}\left(x(t), 0_{\mathbb{I F}}\right)<\epsilon \quad \forall t \geq 0
$$

(2) asymptotically stable, iff $\lim _{t \rightarrow+\infty} d_{\infty}\left(x(t), 0_{\mathbb{I F}}\right)=0$.

Definition 4.4. [15] The solution of (5) is said to be Mittag-Leffler stable if

$$
d_{\infty}\left(x(t), 0_{\mathbb{I F}}\right) \leq\left\{m\left(x_{0}\right) E_{\alpha}\left(-\lambda t^{\alpha}\right)\right\}^{b}
$$

where $\alpha \in[0,1], \lambda \geq 0, b>0, m(0)=0, m(x) \geq 0$ and $m(x)$ is locally Lipschitz on $x$ with Lipschitz constant $m_{0}$.

Remark 4.5. The Mittag-Leffler stability implies the stability of Lyapounov. 


\subsection{Main result}

Theorem 4.6. Let $V(t, x(t)):\left[0,+\infty\left[\times \mathbb{I F}^{1} \rightarrow \mathbb{R}\right.\right.$ be a continuously differentiable function and locally Lipschitz with respect to $x$ such that

$$
\begin{gathered}
c_{1} d_{\infty}^{a}\left(x(t), 0_{\mathbb{I F}}\right) \leq V(t, x(t)) \leq c_{2} d_{\infty}^{a b}\left(x(t), 0_{\mathbb{I F}}\right) \\
{ }^{c} D^{\beta}\left(V(t, x(t)) \leq-c_{3} d_{\infty}^{a b}\left(x(t), 0_{\mathbb{I F}}\right),\right.
\end{gathered}
$$

where $t \geq 0, \beta \in[0,1], c_{1}, c_{2}, c_{3}, a$ and $b$ are positive constants. Then the trivial solution of (5) is Mittag-Leffler stable.

Proof. From equations (6) and (7) we get

$$
{ }^{c} D^{\beta}\left(V(t, x(t)) \leq \frac{-c_{3}}{c_{2}} V(t, x(t)) .\right.
$$

There exists a positive function $k(t)$ satisfying

$$
{ }^{c} D^{\beta}\left(V(t, x(t))+k(t)=\frac{-c_{3}}{c_{2}} V(t, x(t)) .\right.
$$

By using the Laplace transform (1) and (2), we obtain

$$
S^{\beta} L\{V(t, x(t)) ; s\}-S^{\beta-1} V(0, x(0))+L\{k(t) ; s\}=\frac{-c_{3}}{c_{2}} L\{V(t, x(t)) ; s\} .
$$

It follows that

$$
L\{V(t, x(t)) ; s\}=\frac{S^{\beta-1} V(0, x(0))-L\{k(t) ; s\}}{S^{\beta}+\frac{c_{3}}{c_{2}}} .
$$

By using the inverse of Laplace transform we obtain

$$
V(t, x(t))=E_{\beta}\left(\frac{-c_{3}}{c_{2}} t^{\beta}\right) V(0, x(0))-k(t) *\left(t^{1-\beta} E_{\beta, \beta}\left(\frac{-c_{3}}{c_{2}} t^{\beta}\right) .\right.
$$

Since $E_{\beta, \beta}\left(\frac{-c_{3}}{c_{2}} t^{\beta}\right)$ is a nonnegative function [12], it follows that

$$
\begin{aligned}
V(t, x(t)) & \leq E_{\beta}\left(\frac{-c_{3}}{c_{2}} t^{\beta}\right) V(0, x(0)) \\
d_{\infty}\left(x(t), 0_{\mathbb{I F}}\right) & \leq\left[\frac{V(0)}{c_{1}} E_{\beta}\left(\frac{-c_{3}}{c_{2}} t^{\beta}\right)\right]^{\frac{1}{a}},
\end{aligned}
$$

where $V(0)=V(0, x(0))$.

Let $m=\frac{V(0)}{c_{1}}$, then we have

$$
d_{\infty}\left(x(t), 0_{\mathbb{I}}\right) \leq\left[m E_{\beta}\left(\frac{-c_{3}}{c_{2}} t^{\beta}\right)\right]^{\frac{1}{a}},
$$

where $m=0$ if and only if $x(0)=0_{\mathbb{I F}}$.

Since $V(t, x)$ is locally Lipschitz with respect to $x$, it follows that $m=\frac{V(0, x(0))}{c_{1}}$ is Lipschitz with respect to $x(0)$ and $m(0)=0$, wich implies the Mittag-Leffler stability of the problem (5). 


\subsection{Examples}

The following examples are used to prove the stability result.

Example 4.7. Consider the following intuitionistic fuzzy fractional problem:

$$
\left\{\begin{array}{l}
{ }^{c} D^{\beta} x(t)+x(t)=0_{\mathbb{I F}} \\
x(0)=x_{0} \in \mathbb{I F}^{1}
\end{array},\right.
$$

where $0<\beta<1$. We consider the candidate Lipschitz function $V:\left[0,+\infty\left[\times \mathbb{I F}^{1} \rightarrow \mathbb{R}\right.\right.$ such that $V(t, x(t))=d_{\infty}\left(x(t), 0_{\mathbb{I F}}\right)$. We can write that

$$
d_{\infty}\left(x(t), 0_{\mathbb{I F}}\right) \leq V(t, x(t)) \leq d_{\infty}\left(x(t), 0_{\mathbb{I F}}\right)
$$

It follows that $c_{1}=c_{2}=1$.

On the other hand, we have

$$
\begin{aligned}
& { }^{c} D^{\beta}\left(V(t, x(t))={ }^{c} D^{\beta} d_{\infty}\left(x(t), 0_{\mathbb{I F}}\right)=I^{\beta} \frac{d}{d t} d_{\infty}\left(x(t), 0_{\mathbb{I}}\right)\right. \\
& { }^{c} D^{\beta}\left(V(t, x(t)) \leq I^{\beta} d_{\infty}\left(x^{\prime}(t), 0_{\mathbb{I F}}\right)\right. \\
& { }^{c} D^{\beta}\left(V(t, x(t)) \leq d_{\infty}\left(I^{\beta} x^{\prime}(t), 0_{\mathbb{I F}}\right)\right. \\
& { }^{c} D^{\beta}\left(V(t, x(t)) \leq d_{\infty}\left({ }^{c} D^{q} x(t), 0_{\mathbb{I F}}\right)\right. \\
& { }^{c} D^{\beta}\left(V(t, x(t)) \leq d_{\infty}\left((-1) x(t), 0_{\mathbb{I F}}\right)\right. \\
& { }^{c} D^{\beta}\left(V(t, x(t)) \leq-d_{\infty}\left(x(t), 0_{\mathbb{I F}}\right) .\right.
\end{aligned}
$$

Finally, we apply $c_{1}=c_{2}=1$ and $c_{3}=-1$ in Theorem 4.6 , and we obtain

$$
d_{\infty}\left(x(t), 0_{\mathbb{I}}\right) \leq V(0) E_{\beta}\left(-t^{\beta}\right)
$$

where $V(0)=V(0, x(0))=d_{\infty}\left(x_{0}, 0_{\mathbb{I F}}\right)$.

Wich implies the Mittag-Leffler stability of system (8).

Example 4.8. We consider the following intuitionistic fuzzy nonlinear fractional system:

$$
\left\{\begin{array}{l}
{ }^{c} D^{q} x(t)=f(t, x(t)) \\
x(0)=x_{0}
\end{array},\right.
$$

where $0<q<1, x=0_{\mathbb{I F}}$ is the equilibrium point of the problem (9) and $f:\left[0,+\infty\left[\times \mathbb{I} \mathbb{F}^{1} \rightarrow \mathbb{I F}^{1}\right.\right.$ is an intuitionistic fuzzy Lipschitz function whith Lipschitz constant $k>0$.

We suppose that there exists a Lyapunov function $V(t, x(t))$ satisfying the following conditions:

$$
\begin{gathered}
c_{1} d_{\infty}\left(x(t), 0_{\mathbb{I F}}\right) \leq V(t, x(t)) \leq c_{2} d_{\infty}\left(x(t), 0_{\mathbb{I F}}\right) \\
V^{\prime}(t, x) \leq-c_{3} d_{\infty}\left(x, 0_{\mathbb{F}}\right)
\end{gathered}
$$

where $c_{1}, c_{2}, c_{3}$ are positive constants and $V^{\prime}(t, x)=\frac{d V(t, x(t))}{d t}$. 
It follows from (10) and (11) that

$$
{ }^{c} D^{1-q}\left(V(t, x(t))=I^{q} V^{\prime}(t, x) \leq-c_{3} I^{q} d_{\infty}\left(x(t), 0_{\mathbb{I F}}\right) .\right.
$$

Since

$$
d_{\infty}\left(f(t, x), 0_{\mathbb{I F}}\right) \leq k \quad d_{\infty}\left(x(t), 0_{\mathbb{I F}}\right)
$$

then

$$
\begin{aligned}
& { }^{c} D^{1-q}\left(V(t, x(t)) \leq \frac{-c_{3}}{k} I^{q} d_{\infty}\left(f(t, x), 0_{\mathbb{I F}}\right),\right. \\
& { }^{c} D^{1-q}\left(V(t, x(t)) \leq \frac{-c_{3}}{k} d_{\infty}\left(I^{q} f(t, x), 0_{\mathbb{I F}}\right),\right. \\
& { }^{c} D^{1-q}\left(V(t, x(t)) \leq \frac{-c_{3}}{k} d_{\infty}\left(x(t), 0_{\mathbb{I F}}\right) .\right.
\end{aligned}
$$

Finally, by applying $c_{1}, c_{2}$ and $\frac{-c_{3}}{k}$ in Theorem 4.6 , we have

$$
d_{\infty}\left(x(t), 0_{\mathbb{I F}}\right) \leq \frac{V(0)}{c_{1}} E_{1-q}\left(-\frac{c_{3}}{c_{2} k} t^{1-q}\right)
$$

where $V(0)=V(0, x(0))$.

Wich implies the Mittag-Leffler stability of system (9).

\section{Conclusion and future works}

In this paper, we studied the existence and stability results of nonlinear intuitionistic fuzzy fractional-order dynamic systems by using the Schauder fixed point theorem and the notions of Mittag-Leffler stability, and we discussed some sufficient criteria to demonstrate the stability of the trivial solution of the proposed system.

Our future works include the Mittag-Leffler stability of intuitionistic fuzzy multi-variables fractional-order nonlinear systems.

\section{References}

[1] Agarwal, R. P., Lakshmikantham, V., \& Nieto, J. J. (2010). On the concept of solution for fractional differential equations with uncertainty. Nonlinear Analysis: Theory, Methods \& Applications, 72(6), 2859-2862.

[2] Agarwal, R., O’Regan, D., \& Hristova, S. (2015). Stability of Caputo fractional differential equations by Lyapunov functions. Applications of Mathematics, 60, 653-676.

[3] Alikhani, R., \& Bahrami, F. (2013). Global solutions for nonlinear fuzzy fractional integral and integrodifferential equations. Communications in Nonlinear Science and Numerical Simulation, 18(8), 2007-2017. 
[4] Allahviranloo, T., Armand, A., \& Gouyandeh, Z. (2014). Fuzzy fractional differential equations under generalized fuzzy Caputo derivative. Journal of Intelligent and Fuzzy Systems, 26(3), 1481-1490.

[5] Arshad, S., \& Lupulescu, V. (2011). On the fractional differential equations with uncertainty. Nonlinear Analysis: Theory, Methods \& Applications, 74(11), 3685-3693.

[6] Atanassov, K. T. (1986). Intuitionistic fuzzy sets. Fuzzy Sets and Systems, 20(1), 87-96.

[7] Bede, B. (2005). Generalizations of the differentiability of fuzzy-number-valued functions with applications to fuzzy differential equations. Fuzzy Sets and Systems, 151(3), 581-599.

[8] Burton, T. A., \& Zhang, B. (2012). Fractional equations and generalizations of Schaefer's and Krasnoselskii's fixed point theorems. Nonlinear Analysis: Theory, Methods \& Applications, 75(18), 6485-6495.

[9] Diethelm, K., \& Ford, N. J. (2002). Analysis of fractional differential equations. Journal of Mathematical Analysis and Applications, 265(2), 229-248.

[10] Elomari, M., Melliani, S., \& Chadli, L. S. (2016). Solution of intuitionistic fuzzy fractional differential equations. Annals of Fuzzy Mathematics and Informatics, 13(3), 379-391.

[11] Hoa, N. V., Lupulescu, V., \& O’Regan, D. (2018). A note on initial value problems for fractional fuzzy differential equations. Fuzzy Sets and Systems, 347, 54-69.

[12] Kilbas, A., Srivastava, M., \& Trujillo, J. (2006). Theory and Applications of Fractional Differential Equations. North-Holland Mathematical Studies, Vol. 204, Ed van Mill, Amsterdam.

[13] Li, C. P., Zhang, F. R. (2011). A survey on the stability of fractional differential equations. The European Physical Journal Special Topics, 193, 27-47.

[14] Li, Y., Chen, Y. Q., \& Podlubny, I. (2009). Mittag-Leffler stability of fractional order nonlinear dynamic systems. Automatica, 45, 1965-1969.

[15] Li, Y., Chen, Y., \& Podlubny, I. (2010). Lyapunov direct method and generalized MittagLeffler stability. Computers \& Mathematics with Applications, 59(5), 1810-1821.

[16] Liu, K. W., \& Jiang, W. (2011). Finite-time stability of linear fractional order neutral systems. Mathematica Applicata, 24, 724-730.

[17] Matignon, D. (1996). Stability results for fractional differential equations with applications to control processing. In: IMACS-SMC, Lille, France, 963-968.

[18] Melliani, S., Elomari, M., Chadli, L.S., \& Ettoussi, R. (2015). Extension of Hukuhara difference in intuitionistic fuzzy set theory. Notes on Intuitionistic Fuzzy Sets, 21(4), $34-47$. 
[19] Melliani, S., Elomari, M., Chadli, L.S., \& Ettoussi, R. (2015). Intuitionistic fuzzy fractional equation. Notes on Intuitionistic Fuzzy Sets, 21(4), 76-89.

[20] Momani, S., \& Hadid, S. (2004). Lyapunov stability solutions of fractional integrodifferential equations. International Journal of Mathematics and Mathematical Sciences, 47, Article ID 575670, 2503-2507.

[21] Salahshour, S., Allahviranloo, T., Abbasbandy, S., \& Baleanu, D. (2012). Existence and uniqueness results for fractional differential equations with uncertainty. Advances in Difference Equations, 2012, Art. No. 112.

[22] Schauder, J. (1930). Der Fixpunktsatz in Funktionalraümen, Studia Mathematica, 2(1), 171-180.

[23] Schwartz, L. (1997). Analyse I, Theorie des Ensembles et Topologie, Hermann Editeurs, Paris, pp. 346.

[24] Weissinger, J. (1952). Zur Theorie und Anwendung des Iterationsverfahrens. Mathematische Nachrichten, 8(1), 193-212.

[25] Xu, Z. S., \& Yager, R. R. (2006). Some geometric aggregation operators based on intuitionistic fuzzy sets. International Journal of General Systems, 35(4), 417-433.

[26] Zadeh, L. A. (1965). Fuzzy sets. Information and Control, 8(3), 338-356. 